\begin{tabular}{|c|l|}
\hline Title & Advanced functionalization of poly hydroxyal kanoate via the UV -initiated thiol-ene click reaction \\
\hline Author(s) & Tajima, Kenji; Iwamoto, Kosuke; Satoh, Y asuharu; Sakai, Ry osuke; Satoh, Toshifumi; Dairi, Tohru \\
\hline Citation & $\begin{array}{l}\text { A pplied microbiology and biotechnology, 100(10), 4375-4383 } \\
\text { https://doi.org/10.1007/300253-015-7252-3 }\end{array}$ \\
\hline Issue Date & $2016-05$ \\
\hline Doc URL & http://hdl.handle.net/2115/65179 \\
\hline Rights & The final publication is available at link.springer.com \\
\hline Type & article (author version) \\
\hline File Information & AMB_document.pdf \\
\hline
\end{tabular}

Instructions for use 


\section{Advanced functionalization of polyhydroxyalkanoate via the UV-initiated thiol-ene click reaction}

Kenji Tajima*, Kosuke Iwamoto, Yasuharu Satoh, Ryosuke Sakai ${ }^{\dagger}$, Toshifumi Satoh, and Tohru Dairi*

Division of Applied Chemistry, Faculty of Engineering, Hokkaido University, N13W8, Kitaku, Sapporo 060-8628, Japan

*Corresponding author

E-mail address: ktajima@eng.hokudai.ac.jp

Telephone number: +81 11 706-6607

Fax number: +81 11 706-6607

*Corresponding author

E-mail address: dairi@eng.hokudai.ac.jp

Telephone number: +81 11 706-7815

Fax number: +81 11 706-7815

Present address:

'Department of Materials Chemistry, Asahikawa National College of Technology, Asahikawa 071-8142, Japan 


\begin{abstract}
Polyhydroxyalkanoates (PHAs) incorporating vinyl-bearing 3-hydroxyalkanoates were prepared in $8.5-12.9 \mathrm{~g} \cdot \mathrm{L}^{-1}$ yield. The molar ratios $(0-16 \mathrm{~mol} \%)$ of the vinyl-bearing 3hydroxyalkanoate derivatives were controlled by the continuous feeding of undecylenate at various concentrations. Subsequently, the PHAs were functionalized by UV-initiated thiolene click reaction and chemical modification. ${ }^{1} \mathrm{H}$ NMR spectra suggested that 3mercaptopropionic acid and 2-aminoethanthiol were successfully introduced into the vinylbearing PHA. Subsequently, chemical modification using fluorescein or a fibronectin active fragment (GRGDS) was attempted. The former yielded a PHA derivative capable of emitting fluorescence under UV irradiation, which was useful for determining the miscibility of PHA in a composite film comprising poly-L-lactic acid (PLLA) and PHA. In the latter case, PHA bearing GRGDS peptides exhibited cell adhesiveness, suggesting that its biocompatibility was improved upon peptide introduction. Taken together, the UV-initiated thiol-ene click reaction was demonstrated to be useful in PHA modification.
\end{abstract}

Keywords: polyhydroxyalkanoate, medium chain length, click chemistry, double bond, enethiol click reaction 


\section{Introduction}

Polyhydroxyalkanoates (PHAs) are a class of aliphatic polyesters that accumulate in various bacteria under nutrient-limited conditions, acting as carbon and energy storage materials (Chen 2009; Sudesh et al. 2000). To date, PHAs have attracted much attention, as they possess properties similar to common thermoplastics. PHAs are generally produced by in vivo methods, using whole bacterial cells. They are advantageous in that they can also be produced from renewable resources such as sugars and plant oils, and are degraded in the natural environment. However, the applications of poly(3-hydroxybutyrate) $[\mathrm{P}(3 \mathrm{HB})]$, a common PHA produced by bacteria, are limited because of its brittle and stiff nature, and its low functionality, which depend on the PHA monomer compositions.

Monomer compositions of PHAs are mainly determined by the substrate specificities of PHA synthases. PHA synthases, which are key enzymes in PHA biosynthesis, catalyze the polymerization of $(R)$-hydroxyalkanoyl moieties in (R)-hydroxyalkanoyl coenzyme A (HACoA), with the concomitant release of CoA (Rehm 2003; Taguchi and Doi 2004). The HACoA supply pathway to PHA synthases also affects the monomer compositions depending largely on cell metabolic pathways, e.g., de novo fatty acid biosynthesis, its degradation pathways, and on substrate feedings.

Copolymerization with various hydroxyalkanoate (HA) units is an effective method to improve the physical properties and functionalities of PHA, and in this respect, the syntheses of various copolymers have been attempted (Dai et al. 2008; Fukui et al. 1999; Han et al. 2009; Hu et al. 2011; Matsumoto et al. 2011; Matsusaki et al. 1998; Nomura et al. 2004; Page et al. 1992; Shozui et al. 2009; Tajima et al. 2009; Tajima et al. 2012; Tanadchangsaeng et al. 2009; Valentin et al. 2000; Valentin, Reister and Gruys 2000). Recently, the in vivo synthesis of PHA incorporating lactate (LA) was achieved in a single step through the introduction of genes encoding the screened PHA synthase (lactate-polymerizing enzyme) into E. coli 
engineered to accumulate LA-CoA in the cell (Fig. 1, Method 1) (Matsumoto and Taguchi 2010; Shozui et al. 2010; Taguchi et al. 2008; Yamada et al. 2010).

As an alternative approach, we focused our attention on the production of novel PHAs via chemical modification using a click reaction to expand the application range of PHAs (Fig. 1, Method 2). Click reactions are generally high yielding, wide in scope, stereospecific, simple, and produce by-products that can be easily removed without chromatography. Such reactions are widely used as emerging synthetic tools for both high- and low-molecularweight compounds. One representative example of a click reaction is the Huisgen copper(I)catalyzed azide-alkyne 1,3-dipolar cycloaddition (CuAAC), which yields a 1,4-disubstituted five-membered 1,2,3-triazole ring (Kolb et al. 2001). This reaction between azides and alkynes offers high yields and involves functionalities that can be introduced relatively easily in a variety of molecules. In addition to the CuAAc, the thiol-ene reaction is also important in the realm of click chemistry (Carioscia et al. 2007; Hoyle and Bowman 2010, Hoyle et al. 2004; Li et al. 2007). Campos et al. reported the synthesis of various macromolecules via the UV-initiated thiol-ene click reaction, which proceeded quantitatively, with a high degree of specificity in a matter of minutes (Campos et al. 2008; Killops et al. 2008). However, the majority of PHAs studied to date contain no functional groups, and few groups have reported such modification of PHAs (Constantin et al. 1999; Gagnon et al. 1994a, b; Park, Lenz and Goodwin 1998). Thus, to establish a synthetic methodology for novel PHAs, we explore the preparation of PHA bearing vinyl groups (by undecylenate feeding) and their subsequent chemical modifications using a click reaction. 


\section{Materials and methods}

\section{Materials}

Peptone, yeast extract, chloroform, methanol, glycerol, 10-undecenoic acid, potassium dihydrogen phosphate $\left(\mathrm{KH}_{2} \mathrm{PO}_{4}\right)$, diammonium hydrogen phosphate $\left[\left(\mathrm{NH}_{4}\right)_{2} \mathrm{HPO}_{4}\right]$, citric acid, triethylamine, 1-ethyl-3-(3-dimethylaminopropyl) carbodiimide hydrochloride (EDC), and magnesium sulfate heptahydrate $\left(\mathrm{MgSO}_{4} \cdot 7 \mathrm{H}_{2} \mathrm{O}\right)$ were purchased from Wako Pure Chemical Industries (Osaka, Japan). Fluorescein isothiocyanate (FITC), chlorobenzene, and acetone were purchased from Sigma-Aldrich Japan (Tokyo, Japan). 3-Mercaptopropionic acid, 2-aminoethanthiol hydrochloride, and 2,2-dimethoxy-2-phenylacetophenon (DMPA) were purchased from Tokyo Chemical Industry Co., Ltd. (Tokyo, Japan). All other chemicals were of reagent grade or better. Poly-L-lactic acid (PLLA) was also purchased from SigmaAldrich Japan. The fibronectin active fragment (Gly-Arg-Gly-Asp-Ser: GRGDS) was the product of Peptide Institute, Inc. (Osaka, Japan).

\section{Analytical instrumentation and methods}

Polymer composition and accumulation were analyzed using a GC-2010 capillary GC system (Shimadzu Co., Kyoto, Japan) equipped with a flame ionization detector and a Zebron ZB-1 GC capillary column (0.25 mm i.d. × 30 m; Phenomenex Inc., CA, USA). Samples were prepared as follows. Polymer ( 10 mg) or dried microbial cells $(50 \mathrm{mg})$ were treated with a solution of methanol $(1.7 \mathrm{~mL})$, sulfuric acid $(0.3 \mathrm{~mL})$, and chloroform $(2 \mathrm{~mL})$ at $100{ }^{\circ} \mathrm{C}$ for $140 \mathrm{~min}$ to convert the constituents to their methyl esters. Water $(1 \mathrm{~mL})$ was then added to the reaction mixture, and the chloroform layer was used for GC analysis.

The ${ }^{1} \mathrm{H}$ nuclear magnetic resonance (NMR) spectra of the polymers were obtained using a Bruker MSL400 spectrometer (400 MHz; Bruker BioSpin K.K., Yokohama, Japan) at 
$25^{\circ} \mathrm{C}$ and a $90^{\circ}$ pulse, with a $4 \mathrm{~ms}, 3,000 \mathrm{~Hz}$ spectral width and a $4 \mathrm{~s}$ repetition rate. Chemical shifts were reported in ppm using tetramethylsilane (TMS) as an internal reference.

The molecular weights of the obtained polymers were determined by gel permeation chromatography (GPC) using tandem TSKgel Super HZM-H columns (6.0 mm I.D. × $150 \mathrm{~mm}$; TOSOH, Tokyo, Japan) with chloroform as the eluent. Calibration was performed using polystyrene samples as standards.

Ultraviolet (UV) light irradiation of samples was carried out using a UVP Black Ray UV bench lamp XX-15BLB (UVP, LLC, CA, USA).

\section{Media and microorganisms}

Nutrient-rich (NR) medium $\left(10 \mathrm{~g} \cdot \mathrm{L}^{-1}\right.$ peptone, $2 \mathrm{~g} \cdot \mathrm{L}^{-1}$ yeast extract, $10 \mathrm{~g} \cdot \mathrm{L}^{-1}$ meat extract) was used for the preparation of cells for precultivation. MR_Pre medium $\left(22.0 \mathrm{~g} \cdot \mathrm{L}^{-1}\right.$ $\mathrm{KH}_{2} \mathrm{PO}_{4}, 3.0 \mathrm{~g} \cdot \mathrm{L}^{-1}\left(\mathrm{NH}_{4}\right)_{2} \mathrm{HPO}_{4}, 0.80 \mathrm{~g} \cdot \mathrm{L}^{-1}$ citric acid, $20.0 \mathrm{~g} \cdot \mathrm{L}^{-1}$ glycerol) supplemented with $7.0 \mathrm{~mL} \cdot \mathrm{L}^{-1}$ magnesium sulfate solution $\left[20 \%(w / v) \mathrm{MgSO}_{4} \cdot 7 \mathrm{H}_{2} \mathrm{O}\right]$ and $10 \mathrm{~mL} \cdot \mathrm{L}^{-1}$ trace element solution (Kato et al. 1996) was used for precultivation. Pseudomonas putida (P. putida) KT2440 (ATCC 47054) was used for PHA production. MR_Acc medium (3.0 g. $\mathrm{L}^{-1}$ $\left(\mathrm{NH}_{4}\right)_{2} \mathrm{HPO}_{4}, \quad 0.8 \mathrm{~g} \cdot \mathrm{L}^{-1}$ citric acid, $20.0 \mathrm{~g} \cdot \mathrm{L}^{-1}$ glycerol) supplemented with $7.0 \mathrm{~mL} \cdot \mathrm{L}^{-1}$ magnesium sulfate solution [20\%(w/v) $\left.\mathrm{MgSO}_{4} \cdot 7 \mathrm{H}_{2} \mathrm{O}\right], 10 \mathrm{~mL} \cdot \mathrm{L}^{-1}$ trace element solution (Kato et al. 1996), and $6.0 \mathrm{~mL} \cdot \mathrm{L}^{-1}$ antifoaming agent was used for PHA accumulation. The feed solution contained $1500 \mathrm{~g}$ of glycerol and $30 \mathrm{~g}$ of $\mathrm{MgSO}_{4} \cdot 7 \mathrm{H}_{2} \mathrm{O}$ per $3000 \mathrm{~mL}$ of distilled water. $P$. putida was grown at $30^{\circ} \mathrm{C}$ in NR medium. 10 -Undecenoic acid was neutralized by $5 \mathrm{~N} \mathrm{NaOH}$ solution to prepare undecylenate solution ( $\mathrm{pH} 7.0)$.

\section{Preparation of PHA bearing vinyl groups}

A small portion of frozen cells (P. putida KT2440) was placed into NR medium (3 mL) using a platinum loop, and shaking carried out at $30^{\circ} \mathrm{C}$ for $16 \mathrm{~h}$. The overnight culture 
was then inoculated into $\mathrm{NR}$ medium $(27 \mathrm{~mL})$, and incubated at $30{ }^{\circ} \mathrm{C}$ with shaking at 150 strokes $\cdot \mathrm{min}^{-1}$ for $10 \mathrm{~h}$. The culture $(14 \mathrm{~mL})$ was then inoculated into MR_Pre medium (700 mL), and incubated at $30{ }^{\circ} \mathrm{C}$ with shaking at 140 strokes $\cdot \mathrm{min}^{-1}$ for $16 \mathrm{~h}$. The resulting overnight culture was inoculated into MR_Acc medium (3500 mL), and then incubated at $30{ }^{\circ} \mathrm{C}$ rotating at $150 \mathrm{rpm}$ for $72 \mathrm{~h}$ in a $10 \mathrm{~L}$ jar fermenter (BMS-10NP3 DPC-3A; ABLE corporation, Tokyo, Japan). The feed solution (168 mL per shot) containing glycerol and $\mathrm{MgSO}_{4}$ was added to the jar fermenter using the $\mathrm{pH}$-stat method, with an average feeding number of 14 . Undecylenate solution was continuously added to the culture from $16 \mathrm{~h}$ after beginning the cultivation until the end of cultivation. The final concentrations of undecylenate in the media were $0.15,0.30,0.45$, and $0.60 \%(w / v)$.

The collected bacterial cells were washed twice with ethanol and once with distilled water, and then lyophilized. Polymer was extracted from the dried microbial cell bodies using a Soxhlet extractor with chloroform as solvent, and then precipitated by the addition of methanol after the solvent was reduced with an evaporator under reduced pressure at $30^{\circ} \mathrm{C}$. The structure of the prepared PHA bearing vinyl groups (PHAund60) was analyzed by nuclear magnetic resonance (NMR) spectroscopy (Fig. 2a).

\section{General procedure for the UV-initiated thiol-ene click reaction}

The UV-initiated thiol-ene click reaction was performed according to the method described by Campos et al. (Campos et al. 2008). In a vial, the prepared PHA bearing vinyl groups (PHAund60), thiol (10 eq with respect to the vinyl group), and DMPA (0.2 eq) were dissolved in either chlorobenzene or acetone. The vial was sealed with a screw cap, and the solution was purged with argon for 10 min. Irradiation was then applied for $1 \mathrm{~h}$ using a $365 \mathrm{~nm}$ UV lamp (UVP, LLC, CA, USA). Subsequently, the mixture was washed with chloroform/water to remove impurities, and separated into aqueous and organic phases. The 
organic phase was dried over anhydrous $\mathrm{MgSO}_{4}$, and the solvent evaporated to obtain the desired product.

PHAund60- $\mathrm{NH}_{2}$ (2a). 2-Aminoethanethiol and chlorobenzene were used as the thiol compound and solvent, respectively (Fig. 3). In a vial, PHAund60 (1, 1.0 g, 5.90 mmol) was dissolved in dried toluene $(5 \mathrm{~mL})$, and the solvent was evaporated. This operation was repeated twice to remove water from the starting material. 2-Aminoethanethiol $(0.732 \mathrm{~g}$, $9.38 \mathrm{mmol}, 10$ eq with respect to the vinyl group) was dissolved in chlorobenzene (60 $\mathrm{mL})$ and added to the vial. DMPA (48.0 mg, $0.188 \mathrm{mmol}$ ) was then added to the vial, which was subsequently covered with an aluminum foil to block out light. After purging the mixture for 10 min with argon, irradiation with a $365 \mathrm{~nm}$ UV lamp (UVP, LLC, CA, USA) was carried out for $1 \mathrm{~h}$. Following purification by precipitation with methanol, introduction of 2aminoethanethiol to the polymer was confirmed by NMR spectroscopy (Fig. 2b).

PHAund60-COOH (2b). 3-Mercaptopropionic acid was used as the thiol compound, and chlorobenzene was employed as the solvent. In addition, PHAund60 (1, 1.0 g, 5.90 mmol) and 2-aminoethanethiol (1.06 g, $9.38 \mathrm{mmol}, 10 \mathrm{eq}$ with respect to the alkene) were used. Other procedures were the same as that of 2-aminoethanethiol. Following purification, introduction of 3-mercaptopropionic acid to the polymer was confirmed by NMR analysis (Fig. 2c).

\section{Introduction of fluorescein into PHAund60- $\mathrm{NH}_{2}$}

In a vial, PHAund60- $\mathrm{NH}_{2}$ (2a, $1.06 \mathrm{~g}, 6.24 \mathrm{mmol}$ ) was dissolved in dichloromethane (5 mL). Triethylamine (27.4 $\mu \mathrm{L}, 1.0$ eq with respect to the amino group) was added to the vial and stirred at $30{ }^{\circ} \mathrm{C}$ (Fig. 4). FITC (84.9 mg, 1.1 eq with respect to the amino group) was then added, and the resulting mixture was stirred for $2 \mathrm{~h}$. Following this time, the mixture was washed with chloroform/water to remove impurities, and then separated into aqueous and organic phases. The organic phase was dried over anhydrous $\mathrm{MgSO}_{4}$, and the solvent was 
evaporated to obtain the desired product. Introduction of fluorescein into PHA was confirmed by fluorescence spectrophotometry.

\section{Introduction of a fibronectin active fragment (GRGDS) into PHAund60-COOH}

In a vial, PHAund60-COOH (2b, $40 \mathrm{mg}, 0.21 \mathrm{mmol})$ was dissolved in acetone/water (3 mL, 9:1). EDC (6.54 mg, 1.0 eq with respect to the carboxyl group) was added to the vial and stirred at $0{ }^{\circ} \mathrm{C}$ for $15 \mathrm{~min}$ (Fig. 4). The fibronectin active fragment (GRGDS, $184 \mathrm{mg}$, 1.1 eq with respect to the carboxyl group) was then added and the resulting mixture stirred at $30{ }^{\circ} \mathrm{C}$ for $16 \mathrm{~h}$. Then, the mixture was washed with chloroform/water to remove impurities, and was separated into aqueous and organic phases. The organic phase was dried over anhydrous $\mathrm{MgSO}_{4}$, and the solvent evaporated to obtain the desired product. Introduction of the peptides into PHA was confirmed by NMR analysis (Fig. 2d).

\section{Preparation of a composite film composed of PLLA and PHA with fluorescein}

A mixture of PLLA (9.8 g) and PHA bearing fluorescein (3, $0.2 \mathrm{~g}$ ) was dissolved in chloroform (5 mL), and the prepared polymer solution was poured into a PTFE Petri dish. After drying at $30^{\circ} \mathrm{C}$ in air for $16 \mathrm{~h}$, the resulting composite film was observed using a fluorescence microscope (BX40; Olympus Corporation, Tokyo, Japan).

\section{Cell cultivation}

Human Umbilical Vein Endothelial Cells (HUVEC) were used as cells for evaluating the biocompatibility of PHA bearing GRGDS peptides, because HUVEC has $\alpha_{\mathrm{v}} \beta_{3}$ integrin that binds to a RGD peptide. HUVEC were cultured in endothelial basal medium-2 (EBM-2; Lonza Group Ltd., Basel, Switzerland) supplemented with EGM-2, a supplement factor set, at $30{ }^{\circ} \mathrm{C}$ in air containing 5 vol\% $\mathrm{CO}_{2}$. The culture medium was changed every $3 \mathrm{~d}$. At semiconfluent growth, cells were subcultured in the same medium. 
PHAund60-COOH (2b: $100 \mathrm{mg})$ was dissolved in ethanol $(15 \mathrm{~mL})$ and poured into a 24-well plate. EDC and the fibronectin active fragment were then added into the wells, which were incubated at $30{ }^{\circ} \mathrm{C}$ for $16 \mathrm{~h}$. After air-drying at $30{ }^{\circ} \mathrm{C}$ for $24 \mathrm{~h}$, the resulting PHA film was sterilized by UV-irradiation. HUVEC (10,000 cells/well) in EBM-2 medium with EGM-2 were seeded into the 24-wells coated by PHA bearing GRGDS peptides (4) and incubated at $37^{\circ} \mathrm{C}$ in air containing 5 vol\% $\mathrm{CO}_{2}$. Cells were observed using an inverted microscope (IX40; Olympus Corporation).

\section{Results}

\section{Preparation of vinyl-bearing PHAs}

A fed-batch cultivation using the pH-stat method was carried out. To produce PHAs bearing vinyl groups of different molar ratios, a solution of undecylenate was added continuously to the medium using a peristaltic pump. The final undecylenate concentrations in the medium were controlled at $0.15-0.60 \%(w / v)$. The vinyl molar ratios in the polymers reached a peak of $16 \mathrm{~mol} \%$, and were precisely controlled at $0-16 \mathrm{~mol} \%$ (Fig. 5). The conversion ratios of undecylenate to vinyl-bearing 3HA were approximately $22-40 \%$. The yields of the PHAs decreased slightly with an increase in undecylenate concentrations (Table 1 and Fig. 5).

The monomer compositions, molecular weights, and dispersities $(D)$ of the prepared PHAs are listed in Table 2. The contents of the main PHA components, 3-hydroxyoctanoate (3HO) and 3-hydroxydodecanoate (3HD), decreased with an increase in undecylenate concentration. In addition, the contents of the unsaturated 3-hydroxyalkanoates incorporated into the resulting PHAs (i.e., 3-hydroxy-8-nonenoate (C9:1) and 3-hydroxy-10-undecenoate (C11:1)) also increased in the similar manner (Table 2). Furthermore, when the undecylenate concentration reached $0.60 \%(w / v)$, the 3-hydroxy-6-heptenoate (C7:1) unit was detected. The 
molecular weights of the prepared PHAs decreased with an increase in undecylenate concentrations, while their dispersities $(D)$ were relatively constant at all undecylenate concentrations. The result of NMR analysis of the prepared PHAs suggested that the vinyl groups were successfully incorporated into 3HAs (Fig. 2).

\section{UV-initiated thiol-ene click reaction}

Following successful preparation of the vinyl-containing PHAs by fed-batch fermentation, the introduction of amino and carboxyl groups into the PHA was carried out via the UV-initiated thiol-ene click reaction (Fig. 3). PHA (PHAund60) containing 16 mol\% of the vinyl-bearing 3HA was selected as the PHA sample. For introducing amino and carboxyl groups, 2-aminoethanethiol and 3-mercaptopropionic acid were used as thiol compounds, respectively. ${ }^{1} \mathrm{H}$ NMR spectra of the PHAs before and after the click reactions are shown in Fig. 2. Signals corresponding to the vinyl protons were observed at $\sim 4.95$ and $\sim 5.80 \mathrm{ppm}$ in the ${ }^{1} \mathrm{H}$ NMR spectrum before reaction (Fig. 2a). After the thiol-ene click reaction using 3mercaptopropionic acid, these two signals almost completely disappeared (Fig. 2c). In contrast, signals corresponding to the vinyl protons were observed after the reaction with 2aminoethanethiol (Fig. 2b) likely due to the incomplete reaction. The introduction rates of the two thiol compounds (3-mercaptopropionic acid and 2-aminoethanethiol) as estimated from the ${ }^{1} \mathrm{H}$ NMR spectra were $>99 \%$ and $23.0 \%$, respectively. This confirms that the UVinitiated thiol-ene click reaction is applicable for PHA modification, and in particular the introduction of amino and carboxyl groups.

\section{Introduction of fluorescein into $\mathrm{PHAund60- \textrm {NH } _ { 2 }}$}

The functionalization of PHA through the introduction of fluorescein was performed using PHAund60- $\mathrm{NH}_{2}$ (Fig. 4), in which the unit molar ratio of amino groups was $\sim 4 \mathrm{~mol} \%$ [0.16 (unit molar ratio of vinyl groups) $\times 0.23$ (introduction rate of 2-aminoethanethiol) $=$ 


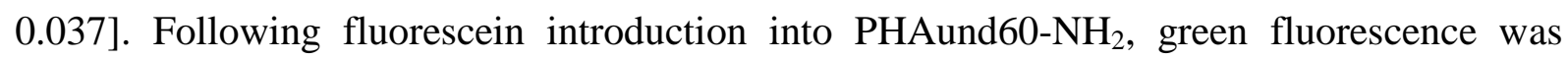
observed upon irradiation with UV light (Fig. 6). The resulting polymer was dissolved in acetone and analyzed by fluorescence spectroscopy. Emission maxima were observed at 480 and $485 \mathrm{~nm}$ for the fluorescein-containing PHAund60 and the free FITC, respectively (Fig. 6). Compared to the free FITC, the maximum emission wavelength for fluorescein-PHA was shifted to a shorter wavelength, likely due to a change in the mobility of fluorescein upon conjugation to PHA through the thiourethane linkage.

A number of PLLA-based polymer alloys including PHA have been reported to reduce the brittleness of PLLA. PHAs produced by the Pseudomonas species are generally mediumchain-length PHAs (mcl-PHAs) that exhibit high elasticity, and are thus effective in reducing PLLA stiffness. As the prepared fluorescein-PHA is capable of emitting fluorescence upon UV-irradiation, its location can be easily determined through fluorescence observations. Therefore, a composite film composed of PLLA and fluorescein-PHA (3) was prepared and observed by fluorescence microscopy. Finely dispersed fluorescein was detected over the entire film (Fig. 7b) in contrast to a composite film composed of PLLA and PHAund60- $\mathrm{NH}_{2}$ (Fig. 7a), confirming that fluorescein-PHA (3) can be used for determining the location of PHA within a composite film.

\section{Introduction of a fibronectin active fragment (GRGDS) into PHAund60-COOH}

PHA functionalization through the introduction of a peptide-based bioactive molecule was also performed using PHAund60-COOH (2b). As a bioactive molecule, the fibronectin active fragment, a GRGDS peptide, was used to improve the biocompatibility of PHA. By ${ }^{1} \mathrm{H}$ NMR analysis, the peaks corresponding to the peptide were observed around 4 ppm (Fig. 2d).

The condensation reaction was first carried out on the film cast on the bottom of a 24well plate to ensure that the GRGDS peptides could be easily contacted with the cells (Fig. 4). Following sterilization by UV-irradiation, HUVEC were placed into the wells, and then 
incubated at $37^{\circ} \mathrm{C}$ under air containing 5 vol\% $\mathrm{CO}_{2}$. The 24-well plate, coated with either GRGDS-PHA or PHAund60-COOH, was monitored with an inverted microscope over $6 \mathrm{~h}$ cultivation. HUVEC adhered on the wells were observed for the 24-well plate coated with the PHA bearing GRGDS peptides (Fig. 8), but not for the 24-well plate coated with only PHAund60-COOH (Fig. 8).

\section{Discussion}

To date, a number of reports on the use of Pseudomonas for the preparation of PHA incorporating unsaturated monomers have been published (Ashby and Foglia 1998; Constantin et al. 1999; Gagnon et al. 1994a, b; Huijberts et al. 1992; Lageveen et al. 198inhi8; Lee et al. 2001; Park, Lenz and Goodwin 1998; Sun et al. 2009). In these studies, various monomers bearing unsaturated units were supplied through $\beta$-oxidation and de novo fatty acid synthetic pathways. In this study, undecylenate, which contains a vinyl group, was used as the source of unsaturated monomer to produce 3HAs bearing a vinyl group at the alkyl side chain terminal (Constantin et al. 1999; Gagnon et al. 1994a, b; Park, Lenz and Goodwin 1998). Because of the toxicity of undecylenate to bacteria cells, it was added gradually to the medium using a peristaltic pump. Using such a feeding method, the molar ratios of 3HAs could be precisely controlled at $0-16 \mathrm{~mol} \%$ (Fig. 5), giving $8.5-12.9 \mathrm{~g} \cdot \mathrm{L}^{-1}$ vinyl-containing PHA in a single cultivation (Table 1 and Fig. 5).

In general PHA production, the monomer units (3HAs) are supplied via a de novo fatty acid synthetic pathway. In contrast, when undecylenate was added, it appeared that 3HAs bearing vinyl groups were supplied by the $\beta$-oxidation pathway based on the following observations: 1) the main components of the PHAs were $3 \mathrm{HO}$ and $3 \mathrm{HD}$, and the contents of vinyl-bearing 3HAs decreased with an increase in undecylenate concentration (Table 2); 2) 3hydroxy-6-heptenoate (C7:1) was also detected in the presence of high undecylenate concentrations (Table 1). 
Copolymerization with various hydroxyalkanoate (HA) units is known to be effective in improving the physical properties and functionalities of PHA, and therefore, the syntheses of various copolymers have been attempted (Dai et al. 2008; Fukui et al. 1999; Han et al. 2009; Hu et al. 2011; Matsumoto et al. 2011; Matsusaki et al. 1998; Nomura et al. 2004; Page et al. 1992; Shozui et al. 2009; Tajima et al. 2009; Tajima et al. 2012; Tanadchangsaeng et al. 2009; Valentin et al. 2000; Valentin, Reister and Gruys 2000). However, few studies have been carried out into the introduction of monomer units bearing amino and carboxyl groups, which exhibit high reactivity and are useful for PHA functionalization. In this study, the amino and carboxyl groups were successfully introduced via a UV-initiated thiol-ene click reaction. The introduction rates of amino and carboxyl groups as estimated from the ${ }^{1} \mathrm{H}$ NMR spectra were $>99 \%$ and $23.0 \%$, respectively (Figs. $2 \mathrm{C}$ and B). Although the introduction rate of amino groups was rather low, the introduced amino groups exhibited high reactivity, and the unit molar ratio ( $\sim$ mol\%) was sufficient for introducing the fluorescent group into PHA (Figs. 6 and 7).

PHAs composed of monomers bearing a side chain of medium chain length (mclPHAs) have been reported to exhibit low glass transition temperatures $\left(\mathrm{T}_{\mathrm{g}}\right)$, and have been used as additives to reduce the stiffness of PLLA (Noda et al. 2004). Even when a small quantity (10-20 wt\%) of mcl-PHA was mixed with PLLA, the properties of PLLA were dramatically improved. Evaluation of the miscibility of components in a polymer alloy is important for understanding the mechanism by which polymer properties are improved. Thus, a composite film composed of PLLA and PHA bearing fluorescein was prepared to determine the location of PHA. As expected, finely dispersed fluorescein was observed over the entire composite film by fluorescence microscopy (Fig. 7), thus confirming the suitability of this method for evaluating PHA dispersibility in a polymer alloy.

In conclusion, we have demonstrated the suitability of the UV-initiated thiol-ene click reaction for the functionalization of PHA. This is a simple and versatile methodology for the 
preparation of functional PHAs, which was achieved via a three-step functionalization process, namely preparation of PHA containing vinyl groups, introduction of amino or carboxyl groups by the UV-initiated thiol-ene click reaction, and functionalization through subsequent chemical modification. A simpler and more versatile development of noble functional PHAs is now in progress in our group and will be reported in the near future. Our work therefore provides a rapid and facile method for evaluating the dispersibility of PHA in a polymer alloy without the requirement for special pre-treatment.

\section{Acknowledgements}

We thank Mr. Eiji Yamada and Mr. Naoya Nakagawa of Hokkaido University for his technical support in NMR measurements. This work was supported by Grant-in-Aid for Scientific Research (No. 21310060 and 21760632) from the Ministry of Education, Culture, Sports, Science, and Technology, Japan.

\section{Compliance with Ethical Standards}

\section{Funding}

This work was supported by Grant-in-Aid for Scientific Research (No. 21310060 and 21760632) from the Ministry of Education, Culture, Sports, Science, and Technology, Japan.

\section{Conflict of interest}

The authors declare that they have no conflict of interest.

\section{Ethical approval}

This article does not contain any studies with human participants or animals performed by any of the authors. 


\section{References}

Ashby RD and Foglia TA (1998) Poly(hydroxyalkanoate) biosynthesis from triglyceride substrates. Appl Microbiol Biotechnol 49:431-437

Campos LM, Killops KL, Sakai R, Paulusse JMJ, Damiron D, Drockenmuller E, Messmore BW, Hawker CJ (2008) Development of thermal and photochemical strategies for thiol-ene click polymer functionalization. Macromolecules 41:7063-7070

Carioscia JA, Schneidewind L, O’brien C, Ely R, Feeser C, Cramer N, Bowman CN (2007) Thiol-norbornene materials: Approaches to develop high $\mathrm{T}_{\mathrm{g}}$ thiol-ene polymers. $\mathrm{J}$ Polym Sci Part A:45 5686-5696

Chen GQ (2009) A microbial polyhydroxyalkanoates (PHA) based bio- and materials industry. Chem Soc Rev 38:2434-2446

Constantin M, Simionescu CI, Carpov A, Samain E, Driguez H (1999) Chemical modification of poly(hydroxyalkanoates). Copolymers bearing pendant sugars. Macromol Rapid Commun 20:91-94

Dai Y, Lambert L, Yuan Z, Keller J (2008) Characterisation of polyhydroxyalkanoate copolymers with controllable four-monomer composition. J Biotechnol 134:137-145

Fukui T, Yokomizo S, Kobayashi G, Doi Y. (1999) Co-expression of polyhydroxyalkanoate synthase and ( $R$ )-enoyl-CoA hydratase genes of Aeromonas caviae establishes copolyester biosynthesis pathway in Escherichia coli. FEMS Microbiol Lett 170:69-75

Gagnon KD, Lenz RW, Farris RJ Chemical modification of bacterial elastomers: 1 . Peroxide crosslinking. (1994a) Polymer 35:4358-4367 
Gagnon KD, Lenz RW, Farris RJ Chemical modification of bacterial elastomers: 2. Sulfur vulcanization. (1994b) Polymer 35:4368-4375

Han X, Satoh Y, Tajima K, Matsushima T, Munekata M (2009) Chemo-enzymatic synthesis of polyhydroxyalkanoate by an improved two-phase reaction system (TPRS). J Biosci Bioeng 108:517-523

Hoyle CE, Bowman CN (2010) Thiol-ene click chemistry. Angew Chem Int Ed 49:15401573

Hoyle CE, Lee TY, Roper T (2004) Thiol-enes: Chemistry of the past with promise for the future. J Polym Sci Part A 42:5301-5338

Hu D, Chung AL, Wu LP, Zhang X, Chen JC, Chen GQ (2011) Biosynthesis and characterization of polyhydroxyalkanoate block copolymer P3HB-b-4HB. Biomacromolecules 12:3166-3173

Huijberts GNM, Eggink G, Waard P, Huisman GW, Witholt B (1992) Pseudomonas putida KT2442 cultivated on glucose accumulates poly(3-hydroxyalkanoates) consisting of saturated and unsaturated monomers. Appl Environ Microbiol 58:536-544

Kato M., Bao HJ, Kang CK, Fukui T., Doi, Y. (1996) Production of a novel copolyester of 3-hydroxybutyric acid and medium chain length 3-hydroxyalkanaic acid by Pseudomonas sp. 61-3 from sugars. Appl Microbiol Biotechnol 45:363-370

Killops KL, Campos LM, Hawker CJ (2008) Robust, efficient, and orthogonal synthesis of dendrimers via thiol-ene “Click” Chemistry. J Am Chem Soc 130:5062-5064

Kolb HC, Finn MG, Sharpless BK (2001) Click chemistry: Diverse chemical function from a few good reactions. Angew Chem Int Ed 40:2004-2021 
Lageveen RG, Huisman GW, Preusting H, Ketelaar P, Eggink G, Witholt B (1988) Formation of polyesters by Pseudomonas oleovorans: Effect of substrates on formation and composition of poly-(R)-3-hydroxyalkanoates and poly-(R)-3hydroxyalkenoates. Appl Environ Microbiol 54:2924-2932

Li Q, Wicks DA, Hoyle CE (2007) Thiourethane-based thiol-ene high $\mathrm{T}_{\mathrm{g}}$ networks: Preparation, thermal, mechanical, and physical properties. J Polym Sci Part A 45:5103-5111

Lee HJ, Choi MH, Kim TU, Yoon SC (2001) Accumulation of polyhydroxyalkanoic acid containing large amounts of unsaturated monomers in Pseudomonas fluorescens BM07 utilizing saccharides and its inhibition by 2-bromooctanoic acid. Appl Environ Microbiol 67:4963-4974

Lowe AB (2014) Thiol-ene “click” reactions and recent applications in polymer and materials synthesis: a first update. Polym Chem 5:4820-4870

Matsumoto K, Taguchi S (2010) Enzymatic and whole-cell synthesis of lactate-containing polyester: toward the complete biological production of polylactate. Appl Microbiol Biotechnol 85:921-932

Matsumoto K, Ishiyama A, Sakai K, Shiba T, Taguchi S (2011) Biosynthesis of glycolatebased polyesters containing medium-chain-length 3-hydroxyalkanoates in recombinant Escherichia coli expressing engineered polyhydroxyalkanoate synthase. J Biotechnol 156:214-217

Matsusaki H, Manji S, Taguchi K, Kato M, Fukui T, Doi Y (1998) Cloning and molecular analysis of the poly(3-hydroxybutyrate) and poly(3-hydroxybutyrate-co-3hydroxyalkanoate) biosynthesis genes in Pseudomonas sp. strain 61-3. J Bacteriol 180:6459-6467 
Noda I, Satkowski MM, Dowrey A., Marcott C (2004) Polymer alloys of Nodax copolymers and Poly(lactic acid). Macromol Biosci 4:269-275

Nomura CT, Taguchi K, Taguchi S, Doi Y (2004) Coexpression of genetically engineered 3-ketoacyl-ACP synthase III ( $f a b H)$ and polyhydroxyalkanoate synthase (phaC) genes leads to short-chain-length-medium-chain-length polyhydroxyalkanoate copolymer production from glucose in Escherichia coli JM109. Appl Environ Microbiol 70:9991007

Page WJ, Manchak J, Rudy B (1992) Formation of poly(hydroxybutyrate-cohydroxyvalerate) by Azotobacter vinelandii UWD. Appl Environ Microbiol 58:28662873

Park WH, Lenz RW, Goodwin S (1998) Epoxidation of bacterial polyesters with unsaturated side chains. I. Production and epoxidation of polyesters from 10undecenoic acid. Macromolecules 31:1480-1486

Rehm BHA (2003) Polyester synthases: natural catalysts for plastics. Biochem J 376:15-33

Shozui F, Matsumoto K, Sasaki T, Taguchi S (2009) Engineering of polyhydroxyalkanoate synthase by Ser477X/Gln481X saturation mutagenesis for efficient production of 3hydroxybutyrate-based copolyesters. Appl Microbiol Biotechnol 84:1117-1124

Shozui F, Matsumoto K, Motohashi R, Yamada M, Taguchi S (2010) Establishment of a metabolic pathway to introduce the 3-hydroxyhexanoate unit into LA-based polyesters via a reverse reaction of $\beta$-oxidation in Escherichia coli LS5218. Polym Degrad Stab 95:1340-1344

Sudesh K, Abe H, Doi Y (2000) Synthesis, structure and properties of polyhydroxyalkanoates: biological polyesters. Prog Polym Sci 25:1503-1555 
Sun Z, Ramsay JA, Guay M, Ramsay BA (2009) Fed-batch production of unsaturated medium-chain-length polyhydroxyalkanoates with controlled composition by Pseudomonas putida KT2440. Appl Microbiol Biotechnol 82:657-662

Taguchi S, Doi Y (2004) Evolution of polyhydroxyalkanoate (PHA) Production system by “enzyme evolution”: successful case studies of directed evolution. Macromol Biosci $4: 145-156$

Taguchi S, Yamada M, Matsumoto K, Tajima K, Satoh Y, Munekata M, Ohno K, Kohda K, Shimamura T, Kambe H, Obata S (2008) A microbial factory for lactate-based polyesters using a lactate-polymerizing enzyme. Proc Natl Acad Sci USA 105:1732317327

Tajima K, Han XR, Satoh Y, Satoh T, Ishii A, Araki Y, Munekata M, Taguchi S (2012) In vitro synthesis of polyhydroxyalkanoate (PHA) incorporating lactate (LA) with a block sequence by using a newly engineered thermostable PHA synthase from Pseudomonas sp. SG4502 with acquired LA-polymerizing activity. Appl Microbiol Biotechnol 94:365-376

Tajima K, Satoh Y, Satoh T, Itoh R, Han XR, Taguchi S, Kakuchi T, Munekata M (2009) Chemo-enzymatic synthesis of poly(lactate-co-(3-hydroxybutyrate)) by a lactatepolymerizing enzyme. Macromolecules 42:1985-1989

Tanadchangsaeng N, Kitagawa A, Yamamoto T, Abe H, Tsuge T (2009) Identification, biosynthesis, and characterization of polyhydroxyalkanoate copolymer consisting of 3-hydroxybutyrate and 3-hydroxy-4-methylvalerate. Biomacromolecules 10:28662874

Valentin HE, Reiser S, Gruys KJ (2000) Poly(3-hydroxybutyrate-co-4-hydroxybutyrate) formation from gamma-aminobutyrate and glutamate. Biotechnol Bioeng 67:291-299 
Valentin HE, Mitsky TA, Mahadeo DA, Tran M, Gruys KJ (2000) Application of a propionyl coenzyme A synthetase for poly(3-hydroxypropionate-co-3hydroxybutyrate) accumulation in recombinant Escherichia coli. Appl Environ Microbiol 66:5253-5258

Yamada M, Matsumono K, Shimizu K, Uramoto S, Nakai T, Shozui F, Taguchi S (2010) Adjustable mutations in lactate (LA)-polymerizing enzyme for the microbial production of LA-based polyesters with tailor-made monomer composition. Biomacromolecules 11:815-819 


\section{Figure Captions}

Fig. 1 Strategies for in vivo production of novel PHAs.

Fig. $2{ }^{1} \mathrm{H}$ NMR spectra of (a) PHAund60, (b) PHAund60- $\mathrm{NH}_{2}$, (c) PHAund60-COOH, and (d) PHAund60-COOH with GRGDS (4). $\mathrm{CDCl}_{3}$ and TMS were used as the solvent and internal standard, respectively. Units A and B shows 3HA and vinyl-bearing 3HA, respectively. $\mathrm{CH}(\mathrm{A} 3$ and $\mathrm{B} 3)$ means methyne protons of units $\mathrm{A}$ and $\mathrm{B}$, and the numbers within the brackets mean the position of the carbon to which the protons bind. $\mathrm{CH}_{2}$ (A2 and B2) means methylene protons of units A and $\mathrm{B} . \mathrm{CH}_{2}\left(\mathrm{~B}^{*}\right)$ means methylene protons adjacent to the vinyl group.

Fig. 3 Introduction of thiol compounds into vinyl-bearing PHA via the thiol-ene click reaction.

Fig. 4 Modification of PHA with either fluorescein or a fibronectin active fragment (a GRGDS peptide).

Fig. 5 PHA yields and unit molar ratios of the vinyl group at various undecylenate concentrations.

Fig. 6 Fluorescence spectra of fluorescein-containing PHA (hollow circles) and free FITC (solid squares). Photographic images of (a) PHAund60- $\mathrm{NH}_{2}$ and (b) Fluoresceincontaining PHA under UV-irradiation.

Fig. 7 Photographic images of the fluorescent microscope observations of composite films, composed of (a) PLLA and PHAund60- $\mathrm{NH}_{2}$ and (b) PLLA and PHA bearing fluorescein.

Fig. 8 Photographic images of light microscope observations of the 24-well plates coated with (a) PHAund60-COOH and (b) PHA containing GRGDS peptides after $6 \mathrm{~h}$ of cultivation.

\section{Table Titles}

Table 1 Cell dry weights, PHA contents, and PHA yields for $P$. putida KT2440 grown under various undecylenate concentrations

Table 2 Monomer compositions and molecular weights of PHAs synthesized using P. putida KT2440 grown under various undecylenate concentrations 
Fig. 1

Method 1: One-step synthesis of new PHAs

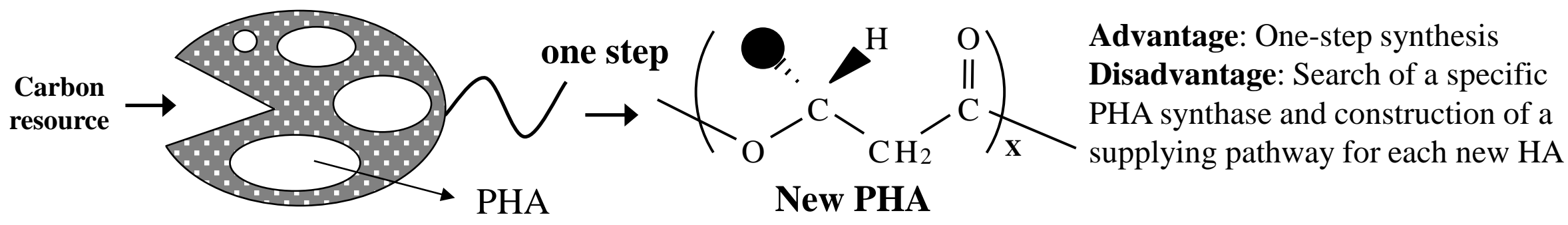

Method 2: Multi-step synthesis of new PHAs
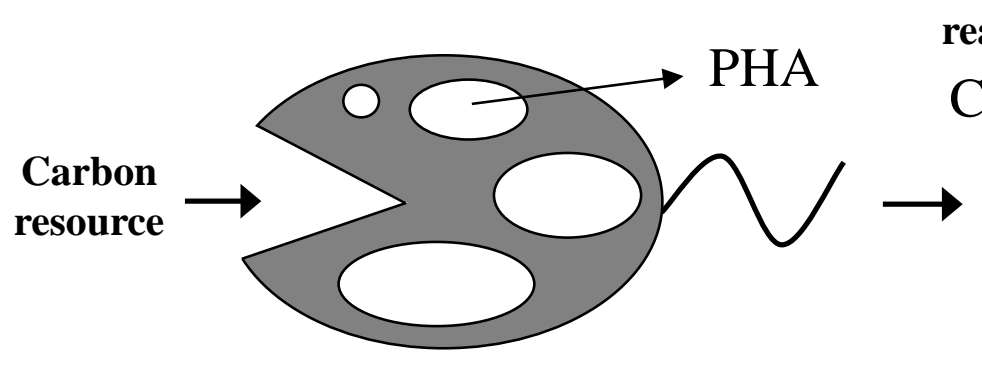

reactive site

Advantage: Availability of synthesis of new PHAs with various structures and functions is possible.

Disadvantage: Multi-step synthesis 
Fig. 2

(a) PHAund60

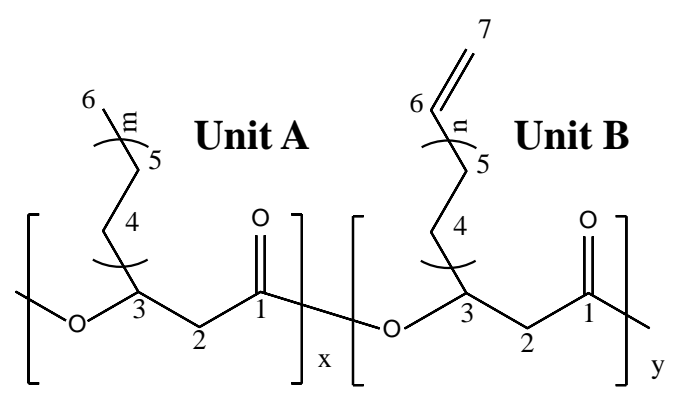

A4, A5,

B6

B7

A2, B2

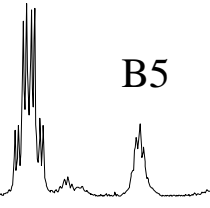

(b) PHAund60-- $\mathrm{NH}_{2}$

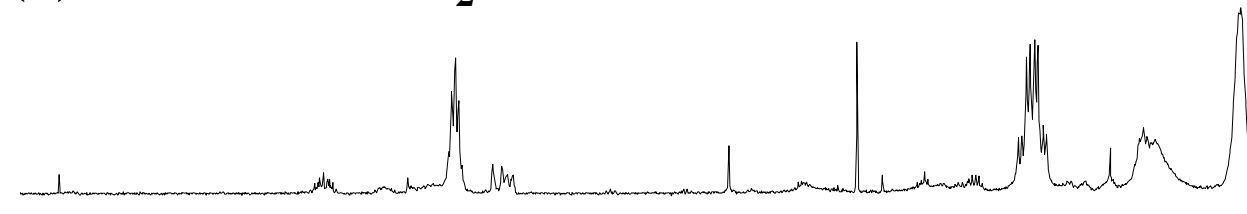

(c) PHAund60-COOH

(d) PHAund60-COOH with GRGDS

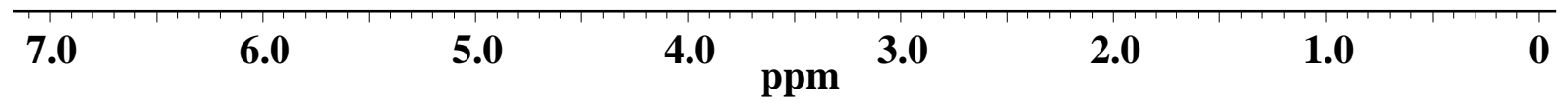


Fig. 3

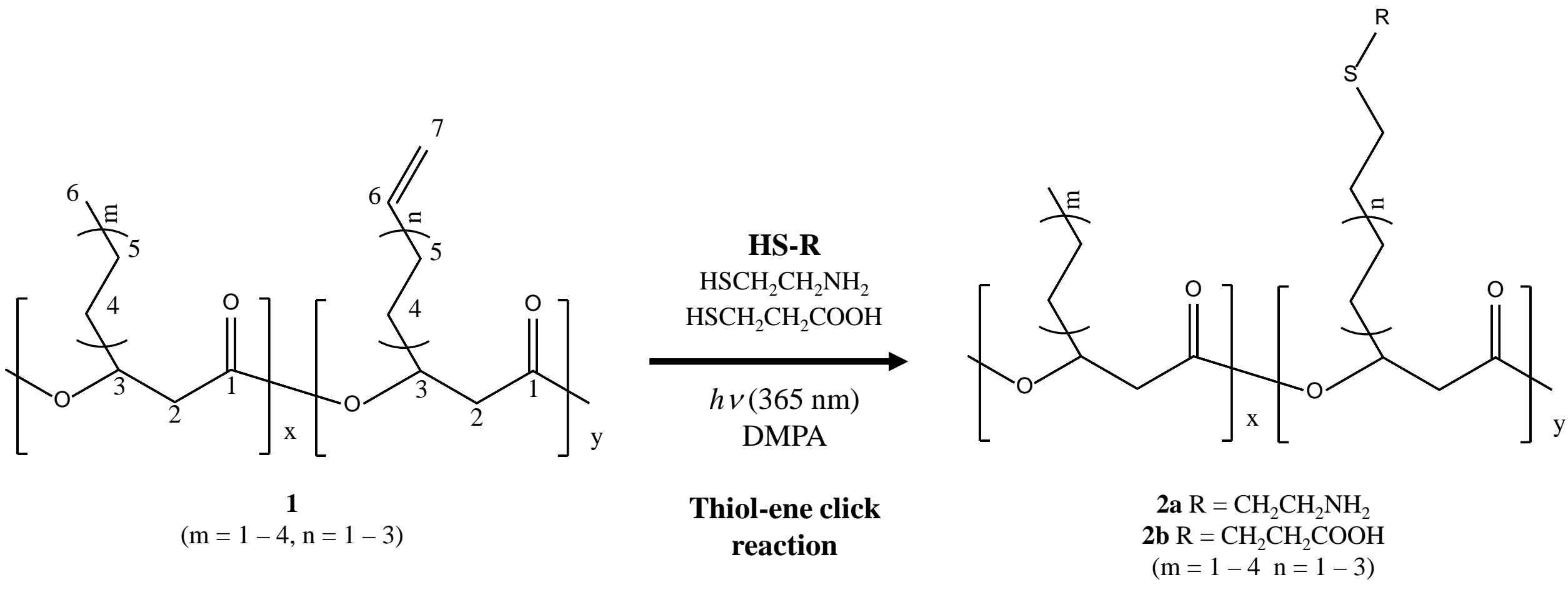


Fig. 4
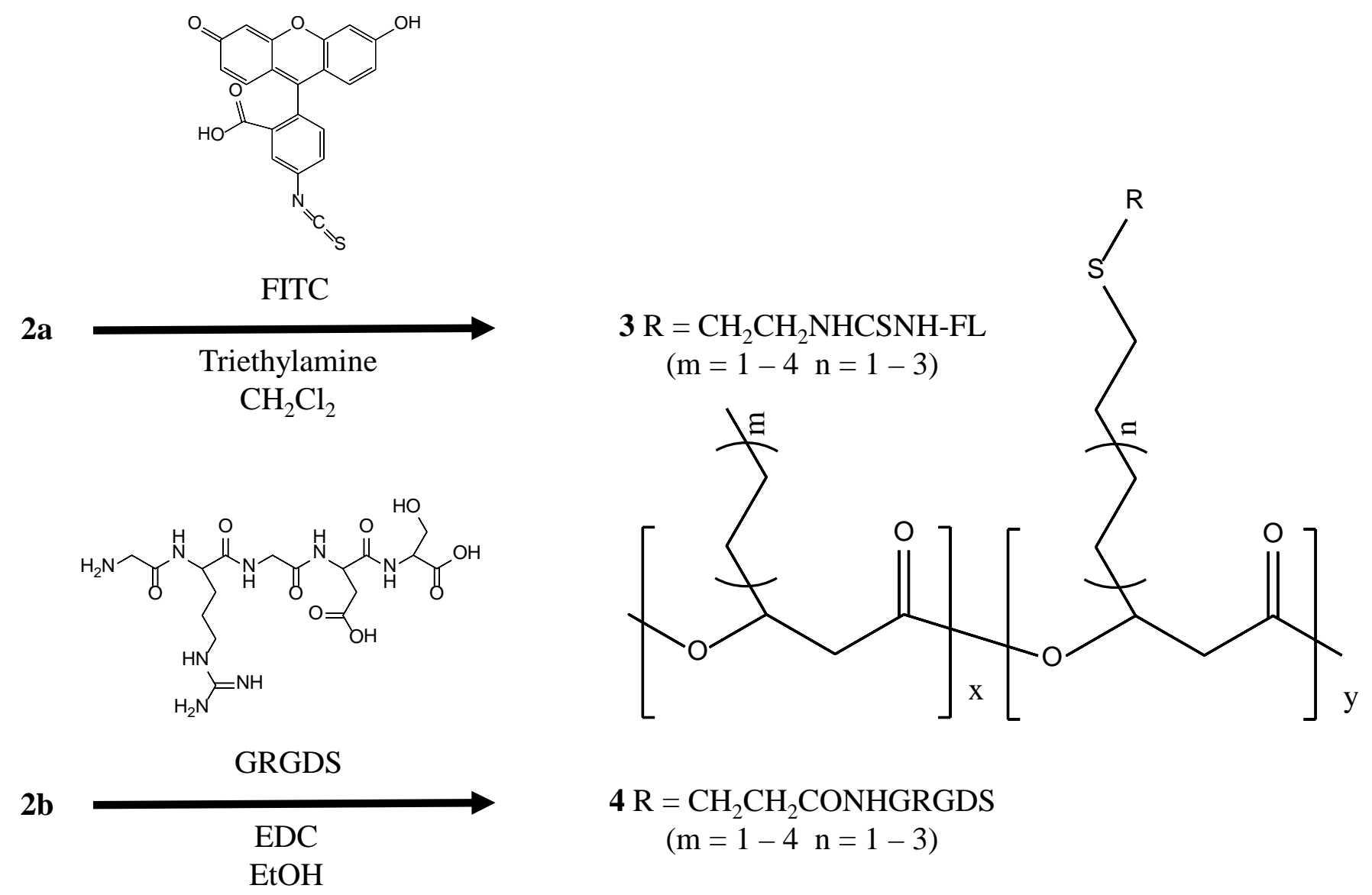
Fig. 5

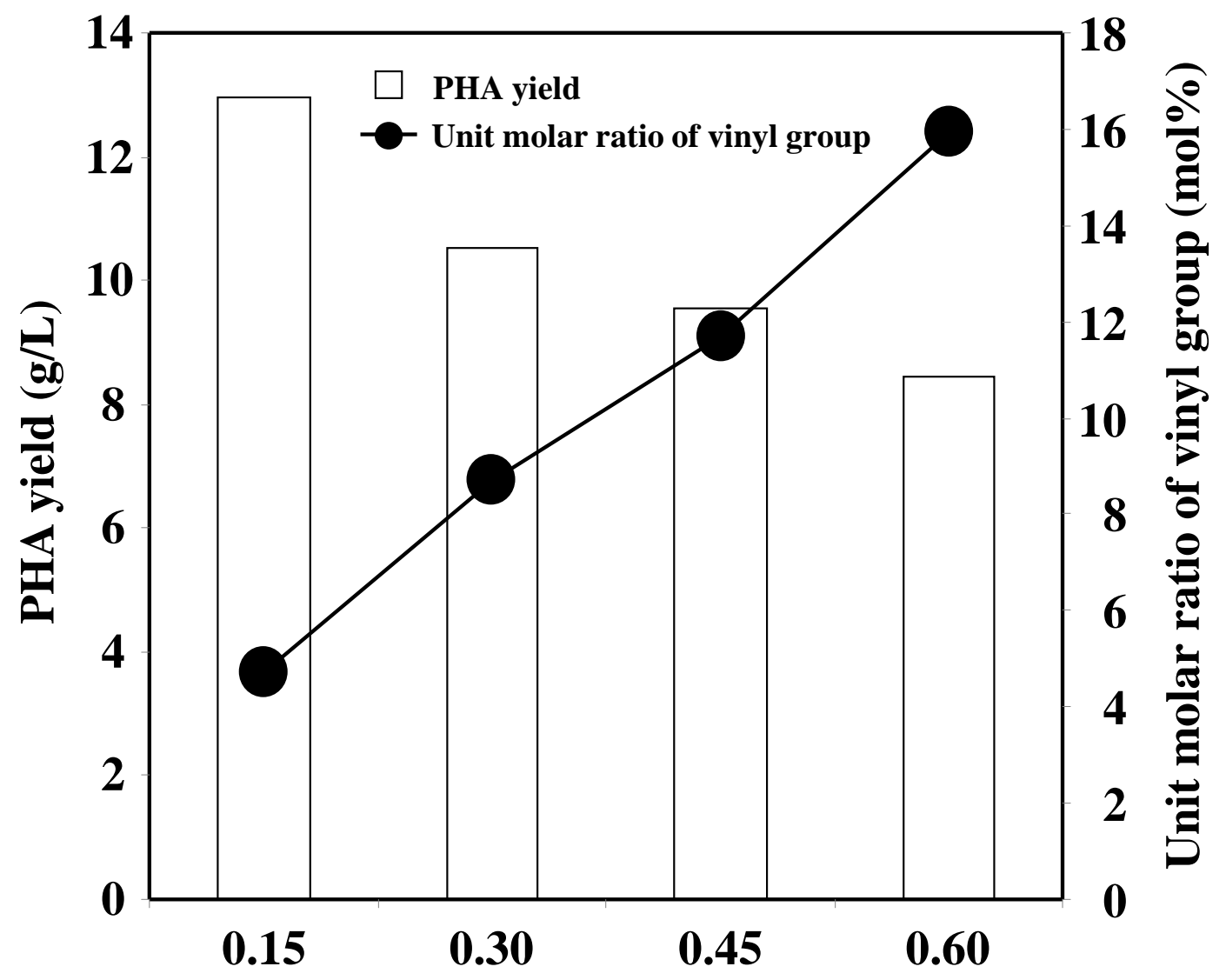

Concentration of undecylenate $[(w / v) \%]$ 
Fig. 6

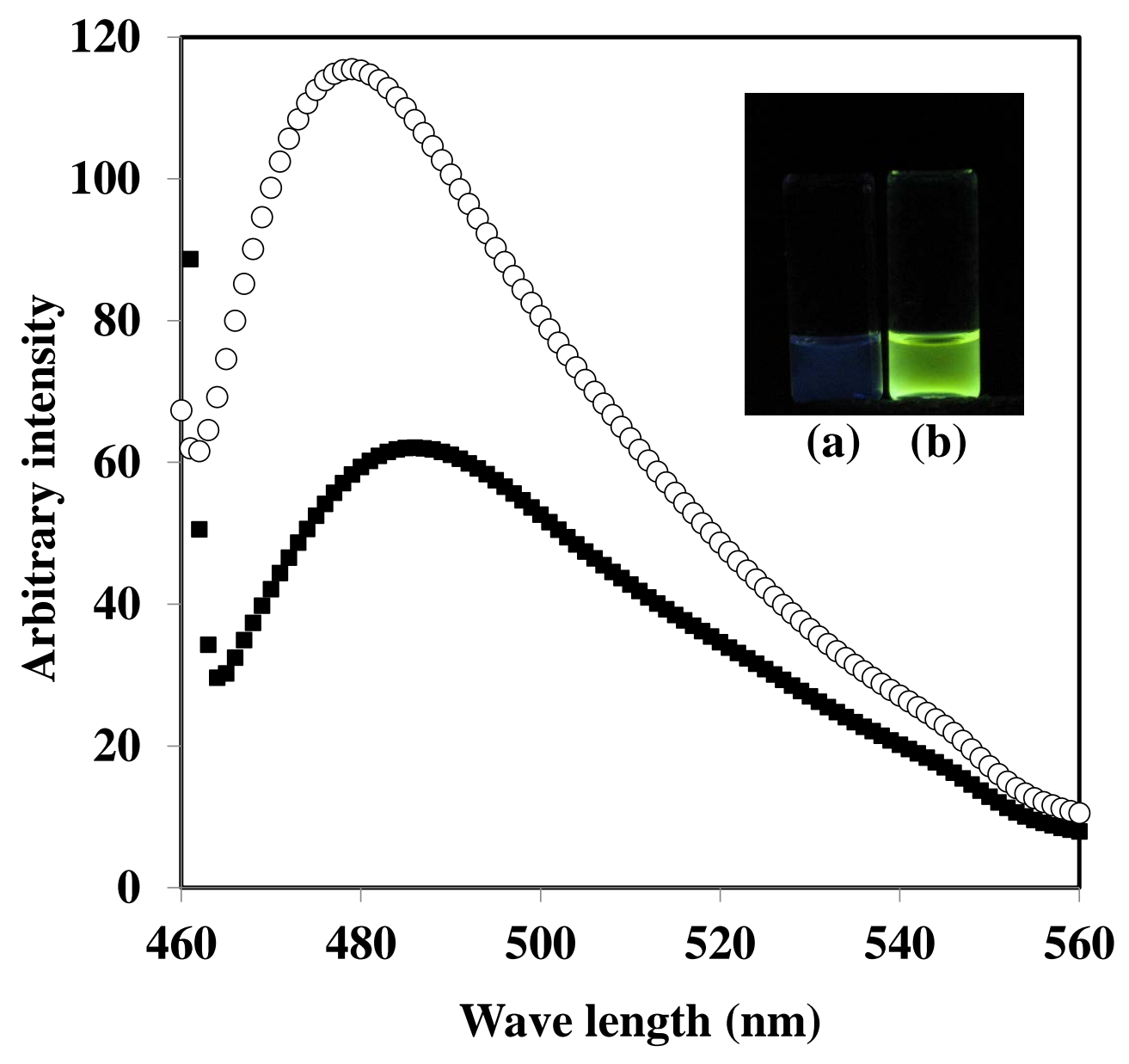


Fig. 7

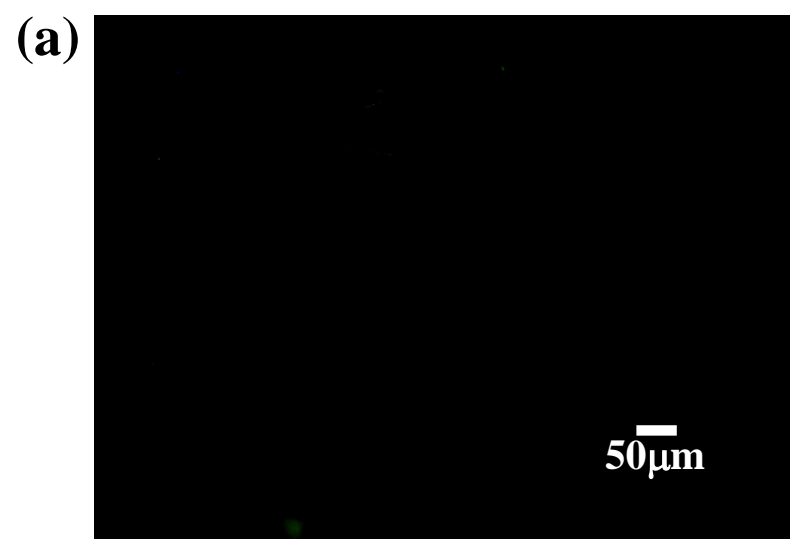

(b)

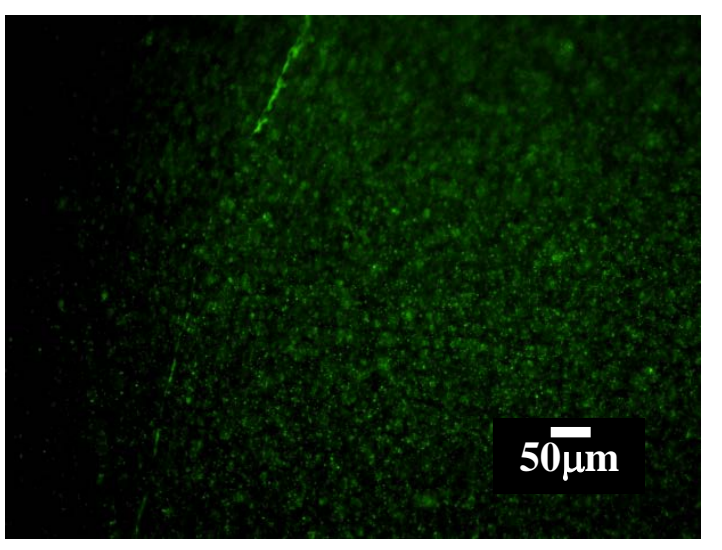


Fig. 8
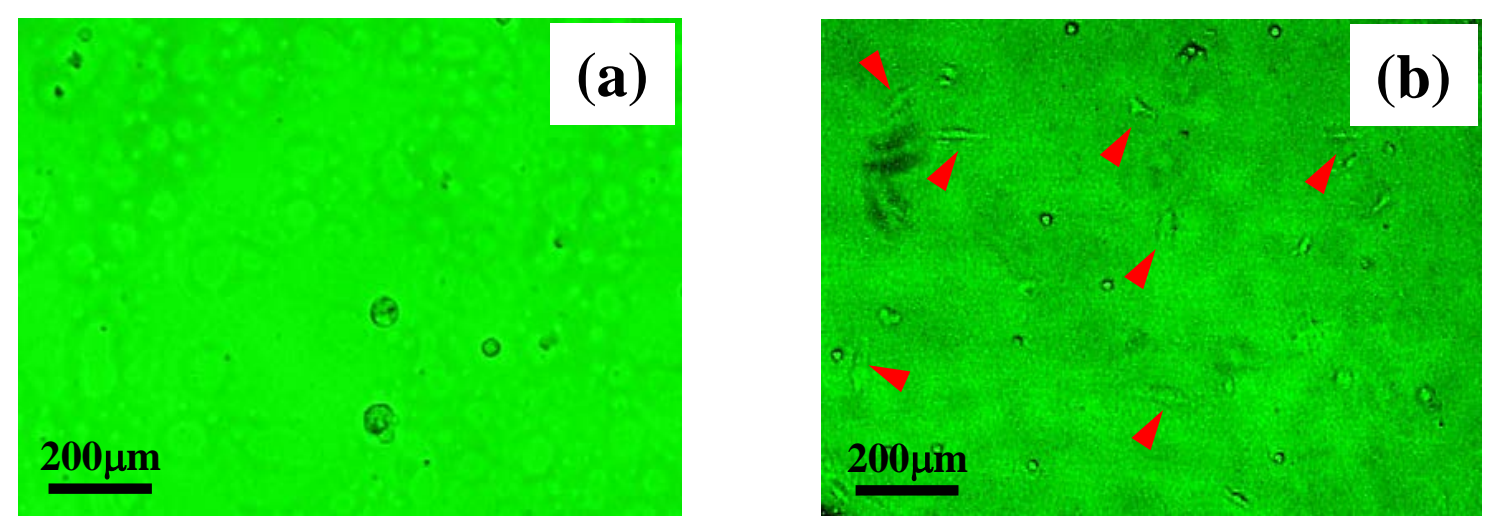
Table 1 Cell dry weights, PHA contents, and PHA yields for P. putida KT2440 grown under various undecylenoate concentrations.

\begin{tabular}{ccccc}
\hline \multirow{2}{*}{ Sample } & $\begin{array}{c}\text { Concentration of undecylenoate } \\
\text { name }\end{array}$ & $\begin{array}{c}\text { Cell dry } \\
\text { weight } \\
\mathrm{g} \mathrm{L}^{-1}\end{array}$ & $\begin{array}{c}\text { PHA content }^{a} \\
\text { (w/v) }\end{array}$ & $\begin{array}{c}\text { PHA yield } \\
\text { L }^{-1}\end{array}$ \\
\hline PHAund15 & 0.15 & 40.5 & 32.0 & 12.9 \\
PHAund30 & 0.30 & 32.5 & 32.4 & 10.5 \\
PHAund45 & 0.45 & 27.5 & 34.7 & 9.5 \\
PHAund60 & 0.60 & 30.2 & 28.0 & 8.5 \\
\hline
\end{tabular}

aDetermined by gas chromatography. 
Table 2 Monomer compositions and molecular weights of PHAs synthesized by P. putida KT2440 grown under various undecylenoate concentrations.

\begin{tabular}{ccccccccccccc}
\hline $\begin{array}{c}\text { Sample } \\
\text { name }\end{array}$ & \multicolumn{1}{c}{ 3HB $^{d}$} & 3HV & 3HHx & C7:1 & 3HO & C9:1 & 3HD & C11:1 & 3HDD & Mw & Mn & $D^{c}$ \\
\hline PHAund0 & 0 & 0 & 1.9 & 0 & 13.0 & 0 & 76.0 & 0 & 7.1 & 58,000 & 30,000 & 1.93 \\
PHAund15 & 0 & 0 & 2.9 & 0 & 17.1 & 1.7 & 67.8 & 3.0 & 7.6 & 54,500 & 28,200 & 1.93 \\
PHAund30 & 0 & 0 & 2.3 & 0 & 13.9 & 2.7 & 67.6 & 6.0 & 7.6 & 49,100 & 26,200 & 1.87 \\
PHAund45 & 0 & 0 & 2.2 & 0 & 18.7 & 5.1 & 60.2 & 6.6 & 7.1 & 32,400 & 15,300 & 2.12 \\
PHAund60 & 0 & 0 & 2.0 & 0.6 & 11.3 & 6.4 & 63.7 & 8.8 & 7.2 & 36,000 & 18,400 & 1.96 \\
\hline
\end{tabular}

aDetermined by gas chromatography.

$b, c$ Determined by GPC using polystyrene samples as standards in chloroform.

${ }^{d}$ 3HB = 3-hydroxybutyrate; 3HV = 3-hydroxyvaryrate; 3HHx = 3-hydroxyhexanoate; C7:1 = 3-hydroxy-6-heptenoate; 3HO = 3-hydroxyoctanoate; C9:1 = 3-hydroxy-8-nonenoate; 3HD = 3-hydroxydecanoate; C11:1 = 3-hydroxy-10-undecenoate; 3HDD = 3-hydroxydodecanoate. 